\title{
NOTAS SÔBRE A PESCA DA CAVALA E DA SERRA NO CEARÁ - DADOS DE $1966^{(1)}$
}

\author{
Raimundo Saraiva da Costa - Melquíades Pinto Paiva
}

\author{
Estação de Biologia Marinha \\ Universidade Federal do Ceará \\ Fortaleza - Ceará — Brasil
}

Com o presente trabalho, a Estação de Biologia Marinha da Universidade Federal do Ceará dá prosseguimento à série de estudos sôbre a pesca da cavala, Scomberomorus cavalla (Cuvier, 1829), e da serra, Scomberomorus maculatus (Mitchill, 1815), efetuada em águas costeiras do Estado do Ceará, Brasil.

Ressaltamos que durante o ano de 1966 a pesca dêsses peixes foi conduzida dentro do padrão tradicional, com embarcações, artes e métodos pesqueiros primitivos.

Os dados dêste trabalho foram obtidos na mesma área dos anteriores (Costa \& Paiva, $1963,1964,1965$ e 1966), e obedecem à mesma orientação metodológica. Mais uma vez fizemos o contrôle de pescarias, tendo em vista, principalmente, o conhecimento da densidade relativa da cavala e da serra, na área em estudo.

\section{A T E R I A L}

O material que serve de fundamento a êste trabalho foi capturado em frente ao município de Fortaleza, tendo sido desembarcado na Praia de Mucuripe (Colônia de Pesca $\mathrm{Z}-20$ ) . Corresponde a amostras de 234 dias de pescaria, abrangendo os meses de janeiro a dezembro de 1966 . As pescarias foram realizadas por jangadas e alguns botes motorizados, operando com linhas de corso e iscas de sardinhas, principalmente. Foram amostradas 2.665 cavalas e 3.178 serras, num total de 5.843 indivíduos (tabela I). Também controlamos 1.551 pescarias de jangadas, realizadas dentro do padrão referido, na mesma área e durante os meses de janeiro a dezembro de 1966 (tabela VIII) .

(1) - Trabalho realizado em decorrência do convênio celebrado com a Superintendência do Desenvolvimento do Nordeste (SUDENE).

\section{T O D O}

Em cada amostra procuramos incluir o máximo possível de indivíduos de ambas as espécies, desembarcados na praia de Mucuripe, e registrados à tarde, após a chegada das embarcações que volviam da faina diária de pesca.

Durante a obtenção das amostras, para cada indivíduo, anotamos apenas a espécie e o comprimento zoológico. Por serem espécimens de alto valor comercial e de demanda imediata, o conhecimento dos sexos estava acima das nossas possibilidades, uma vez que eram vendidos antes mesmo da evisceração.

A identificação das espécies foi efetuada por meio de chave sistemática para a família Scombridae, baseada nos trabalhos de Fraser - Brunner (1950) e Rivas (1951) .

Consideramos como comprimento zoológico (fork length) a distância entre o extremo anterior da cabeça e o extremo posterior dos raios medianos da caudal. estando o animal estendido lateralmente sôbre uma superfície plana.

O contrôle das pescarias foi efetuado diàriamente, na praia de Mucuripe, logo após a chegada das embarcações selecionadas.

De cada pescaria realizada anotamos, principalmente, o número de indivíduos capturados de cavala e de serra, o número de anzóis empregados e o tipo de isca usado, cujos dados foram agrupados por mês.

Os índices de densidade relativa, determinados isoladamente para cada espécie e para ambas em conjunto, se baseiam nos totais dos indivíduos mensalmente capturados por centena de anzóis/dia empregados, em cada mês do período considerado.

Os dados de pluviosidade nos foram fornecidos pela Estação Meteorológica de FortaIeza, do Serviço Meteorológico do Ministério da Agricultura do Brasil. 
T A B E L A I

Número de amostras e de indivíduos amostrados, correspondentes a pescarias de cavala, Scomberomorus cavalla (Cuvier, 1829), e de serra, Scomberomorus maculatus (Mitchill, 1815), realizadas em frente ao município de Fortaleza (Estado do Ceará, Brasil), durante os meses de janeiro a dezembro de 1966.

\begin{tabular}{|c|c|c|c|c|}
\hline \multirow{2}{*}{ meses } & \multirow{2}{*}{$\begin{array}{l}\text { número de } \\
\text { amostras }\end{array}$} & \multicolumn{3}{|c|}{ número de indivíduos } \\
\hline & & cavala & serra & total \\
\hline $\begin{array}{l}\text { janeiro } \\
\text { fevereiro } \\
\text { março } \\
\text { abril } \\
\text { maio } \\
\text { junho } \\
\text { julho } \\
\text { agôsto } \\
\text { setembro } \\
\text { outubro } \\
\text { novembro } \\
\text { dezembro } \\
\end{array}$ & $\begin{array}{l}19 \\
15 \\
23 \\
16 \\
20 \\
20 \\
19 \\
22 \\
21 \\
19 \\
19 \\
21 \\
\end{array}$ & $\begin{array}{r}270 \\
121 \\
283 \\
181 \\
182 \\
230 \\
178 \\
339 \\
189 \\
82 \\
204 \\
406 \\
\end{array}$ & $\begin{array}{r}43 \\
12 \\
30 \\
25 \\
25 \\
47 \\
24 \\
71 \\
620 \\
1.130 \\
555 \\
596 \\
\end{array}$ & $\begin{array}{r}313 \\
133 \\
313 \\
206 \\
207 \\
277 \\
202 \\
410 \\
809 \\
1.212 \\
759 \\
1.003 \\
\end{array}$ \\
\hline total & 234 & 2.665 & 3.178 & 5.843 \\
\hline
\end{tabular}

\section{DISCUSSÃO E CONCLUSÕES}

Das três espécies do gênero Scomberomorus Lacépède, 1802 , que ocorrem em águas costeiras do Brasil (Fowler, 1941) , apenas não registramos a espécie Scomberomorus regalis (Bloch, 1793), apesar da mesma ser também encontrada em águas costeiras do Estado do Ceará (Costa \& Paiva, 1963) .

$\mathrm{Na}$ área e espaço de tempo estudados, a serra foi mais atingida pela pesca do que a cavala nas amostras realizadas (tabela I), verificando-se o contrário nas pescarias controladas (tabela VIII) .

Nos conjuntos mensais de amostras, a cavala apresentou, no período de janeiro a agôsto, freqüências absolutas superiores às anotadas para a serra (tabela I) .

Para a cavala, os tamanhos atingidos pela pesca, no conjunto geral das amostras, variaram entre 35,0 a $135,0 \mathrm{~cm}$ (correspondendo a indivíduos com II a mais de XII anos de idade, de acôrdo com Nomura \& Rodrigues, 1967) , concentrando-se entre 50,0 e $95,0 \mathrm{~cm}$, com máximo entre 60,0 e $65,0 \mathrm{~cm}$ de comprimento zoológico (tabelas II e III ; figura 1). Para a serra, êstes tamanhos variaram entre 30,0 e $110,0 \mathrm{~cm}$ (correspondendo a indivíduos com II a mais de $\mathrm{X}$ anos de idade, de acôrdo com Nomura, 1967), concentrando-se entre 35,0 e $60,0 \mathrm{~cm}$, com máximo entre 40,0 e

T A B E L A I I

Freqüências absolutas de cavala, Scomberomorus cavalla (Cuvier, 1829), por classes de comprimento zoologico $(\mathrm{cm})$, por meses e no total das amostras. Material capturado em frente ao município de Fortaleza (Estado do Ceará - Brasil), durante os meses de janeiro a dezembro de 1966 .

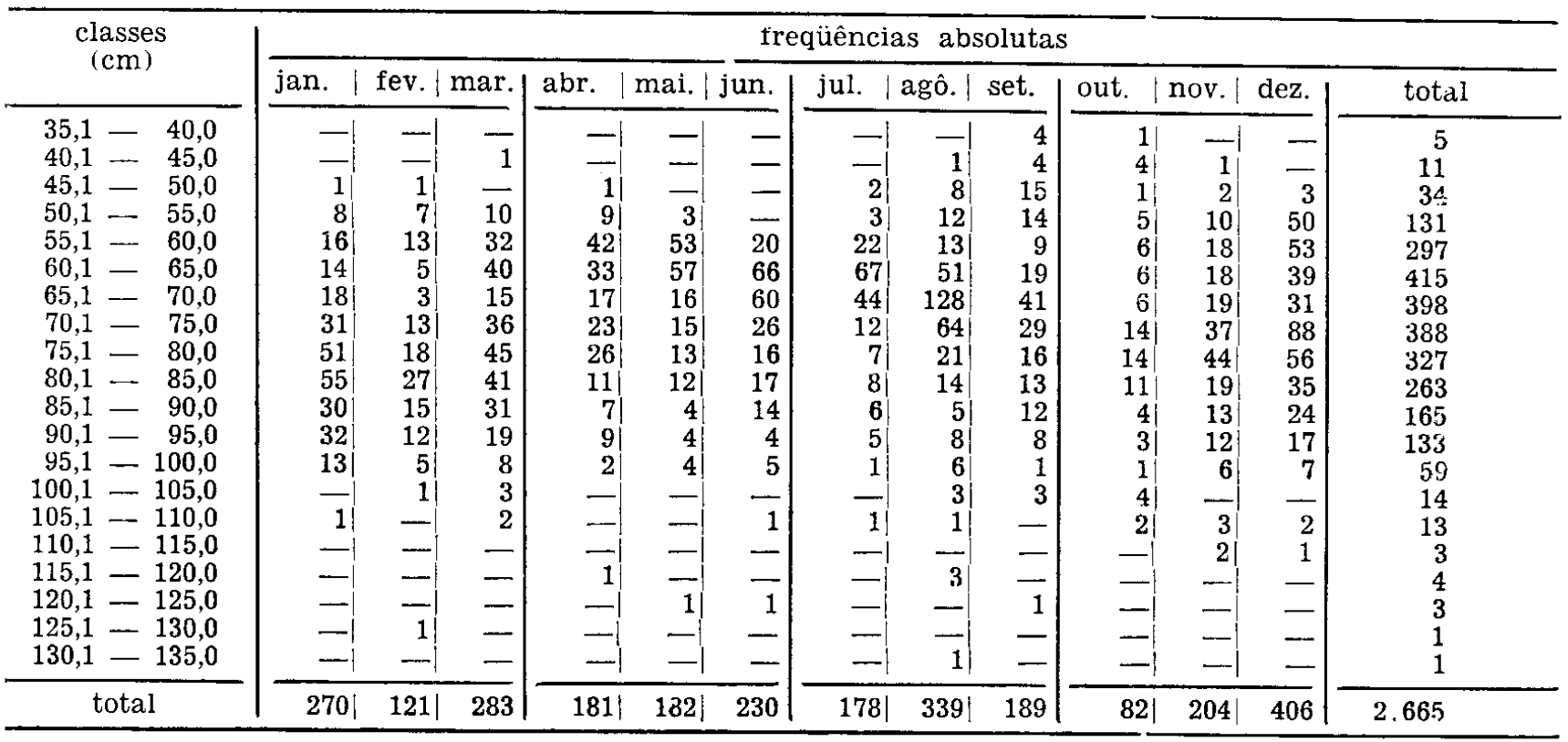


T A B E L A I I I

Freqüências relativas de cavala, Scomberomorus cavalla (Cuvier, 1829), por classes de comprimento zoologico $(\mathrm{cm})$, por meses e no total das amostras. Material capturado em frente ao município de Fortaleza (Estado do Ceará - Brasil), durante os meses de janeiro a dezembro de 1966.

\begin{tabular}{|c|c|c|c|c|c|c|c|c|c|c|c|c|c|c|}
\hline \multirow{2}{*}{\multicolumn{2}{|c|}{$\begin{array}{c}\text { classes } \\
(\mathrm{cm})\end{array}$}} & \multicolumn{13}{|c|}{ freqüências relativas } \\
\hline & & \multirow{2}{*}{$\frac{\operatorname{san} .1}{-1}$} & fev. 1 & mar. & abr. & mai. | & jun. & \multicolumn{2}{|c|}{ jul. | agô. } & \multirow{2}{*}{ set. } & \multirow{2}{*}{$\frac{\text { out. }}{0,04 \mid}$} & \multicolumn{2}{|c|}{ nov. | dez. } & \multirow{2}{*}{$\frac{\text { total }}{0,19}$} \\
\hline 35,1 & 40,0 & & -1 & - & -1 & $--\mid$ & - & -1 & -1 & & & 一 & - & \\
\hline 40,1 & 45,0 & -1 & -1 & 0,04 & -1 & -1 & - & -1 & 0.04 & 0,15 & 0,15 & 0,04 & $\cdots$ & 0,42 \\
\hline 45,1 & 50,0 & 0,04 & 0,04 & - & 0,04 & -1 & - & 0,08 & 0,30 & 0,56 & 0,04 & 0,08 & 0,11 & 1,29 \\
\hline 50,1 & 55,0 & 0,30 & 0,26 & 0,38 & 0,34 & 0,11 & - & 0,11 & 0,45 & 0,53 & 0,18 & 0,38 & 1,88 & 4,92 \\
\hline 55,1 & 60,0 & 0,60 & 0,49 & 1,20 & 1,58 & 1,99 & 0,75 & 0,83 & 0,49 & 0,34 & 0,22 & 0,68 & 1,99 & 11,16 \\
\hline 60,1 & 65,0 & 0,53 & 0,18 & 1,50 & 1,24 & 2,14 & 2,47 & 2,50 & 1,91 & 0,71 & 0,22 & 0,68 & 1,46 & 15,54 \\
\hline 65,1 & 70,0 & 0,68 & 0,11 & 0,56 & 0,64 & 0,60 & 2,25 & 1,65 & 4,80 & 1,54 & 0,22 & 0,71 & 1,16 & 14,92 \\
\hline 70,1 & 75,0 & 1,16 & 0,49 & 1,35 & 0,86 & 0,56 & 0,98 & 0,45 & $\therefore, 40$ & 1,09 & 0,53 & 1,39 & 3,30 & 14,56 \\
\hline 75,1 & 80,0 & 1,31 & 0,68 & 1,69 & 0,98 & 0,49 & 0,60 & 0,26 & 0,79 & 0,60 & 0,53 & 1,65 & 2,10 & 12,28 \\
\hline 80,1 & 85,0 & 2,06 & 1,01 & 1,54 & 0,41 & 0,45 & 0,64 & 0.30 & 0,53 & 0,49 & 0,41 & 0,71 & 1,31 & 9,86 \\
\hline 85,1 & 90,0 & $?, 13$ & 0,56 & 1,16 & 0,26 & 0,15 & 0,53 & 0,22 & 0,18 & 0.45 & 0,15 & 0,49 & 0,90 & 6,18 \\
\hline 90,1 & 95,0 & 1,20 & 0,45 & 0,71 & 0,34 & 0,15 & 0,15 & 0,18 & 0,30 & 0,30 & 0,11 & 0,45 & 0,64 & 4,98 \\
\hline 95,1 & 100,0 & 0,49 & 0,18 & 0,37 & 0,08 & 0,15 & 0,18 & 0,04 & 0,22 & 0,04 & 0,04 & 0,22 & 0,26 & 2,20 \\
\hline 100,1 & 105,0 & -1 & 0,04 & 0.11 & -1 & -1 & - & - & 0,11 & 0,11 & 0,15 & - & - & 0,52 \\
\hline 105,1 & 110,0 & 0,04 & -1 & 0,08 & -1 & --1 & 0,04 & 0,04 & $\mathrm{C}, 04$ & - & 0,08 & 0,11 & 0,08 & 0,51 \\
\hline 110,1 & 115,0 & -1 & -1 & - & -1 & -1 & - & -1 & -1 & - & - & 0,08 & 0,04 & 0,12 \\
\hline 115,1 & 120,0 & -1 & -1 & - & 0,04 & -1 & - & $-!$ & 0,11 & - & -1 & -1 & - & 0,15 \\
\hline 120,1 & 125,0 & -1 & -1 & - & -1 & 0,04 & 0,04 & -1 & -1 & 0,04 & - & -1 & - & 0,12 \\
\hline 125,1 & 130,0 & -1 & $C, 04 \mid$ & - & - & - & - & -1 & -1 & - & -1 & - & - & 0,04 \\
\hline 130,1 & 135,0 & -1 & -1 & -- & -1 & -1 & - & -1 & 0,04 & - & -1 & -1 & - & 0,04 \\
\hline & & $10.14 \mid$ & 4,53 & 10,62 & $6,81 \mid$ & $6,83 \mid$ & 8,63 & 6.66 & $2,71 \mid$ & 7,10 & $3,07 \mid$ & 7,67 & $\overline{15,23}$ & 100,00 \\
\hline
\end{tabular}

T A B E L A I V

Freqüências absolutas de serra, Scomberomorus maculatus (Mitchill, 1815), por classes de comprimento zoológico $(\mathrm{cm})$, por meses e no total das amostras. Material capturado em frente ao município de Fortaleza (Estado do Ceará - Brasil), durante os meses de janeiro a dezembro de 1966.

\begin{tabular}{|c|c|c|c|c|c|c|c|c|c|c|c|c|c|c|}
\hline \multirow{2}{*}{\multicolumn{2}{|c|}{$\begin{array}{c}\text { classes } \\
(\mathrm{cm})\end{array}$}} & \multicolumn{13}{|c|}{ freqüências absolutas } \\
\hline & & \multicolumn{3}{|c|}{ jan. | fev. | mar. } & \multicolumn{3}{|c|}{ abr. | mai. | jun. } & \multicolumn{3}{|c|}{ jul. | agô.| set. } & \multicolumn{3}{|c|}{ out. | nov. | dez. } & \multirow{2}{*}{$\begin{array}{r}\text { total } \\
6\end{array}$} \\
\hline $30,1-$ & 35,0 & -1 & -1 & - & 3 & - & - & -1 & -1 & 1 & 1) & $1)$ & - & \\
\hline $35,1-$ & 40,0 & -1 & -1 & 1 & $3 \mid$ & -1 & 1 & 2 & 10 & 74 & 77 & 22 & 3 & 193 \\
\hline 40,1 & 45,0 & $\mathbf{5}$ & -1 & 2 & 6 & -1 & $\ldots$ & 3 & 7 & 173 & 415 & 186 & 200 & 997 \\
\hline $45,1-$ & 50,0 & 3 & 3 & 3 & $2 \mid$ & 7 & 6 & 6 & 13 & 169 & 374 & 146 & 206 & 938 \\
\hline $50,1-$ & 55,0 & 3 & 4 & 11 & 3 & 4 & 5 & $4 !$ & 20 & 107 & 155 & 92 & 107 & 515 \\
\hline $55,1-$ & 60,0 & 3 & 31 & 8 & $4 \mid$ & 6 & 6 & $4 !$ & 13 & 63 & 511 & 52 & 45 & 258 \\
\hline $60,1-$ & 65,0 & 10 & 1 & 1 & -1 & $1 \mid$ & 14 & $1 \mid$ & 5 & 23 & 281 & 221 & 20 & 126 \\
\hline $65,1-$ & 70,0 & 8 & 1 & 2 & 2 & 5 & 8 & 3 & 21 & 5 & 10 & 16 & 7 & 69 \\
\hline $70,1-$ & 75,0 & 71 & -1 & 1 & $1 \mid$ & 1 & 6 & -1 & $1 \mid$ & 4 & 11 & 11 & 7 & 50 \\
\hline $75,1-$ & 80,0 & 3 & -1 & 1 & 11 & 1 & 1 & -1 & -1 & 1 & 6 & 5 & - & 19 \\
\hline $80,1-$ & 85,0 & 1 & -1 & - & -1 & - & - & -1 & -1 & 二 & -1 & $2 \mid$ & - & 3 \\
\hline $85,1-$ & 90,0 & -1 & -1 & - & -1 & -1 & - & 1 & -1 & - & 1 & -1 & 1 & 3 \\
\hline $90,1 \ldots$ & 95,0 & -1 & -1 & - & -1 & -1 & 一 & -1 & -1 & - & -1 & -1 & - & - \\
\hline $95,1-1$ & 100,0 & -1 & -1 & - & -1 & -1 & - & -1 & -1 & - & -1 & -1 & - & - \\
\hline $100,1-1$ & 105,0 & -1 & -1 & - & -1 & -1 & 一 & -! & - & - & 1) & -1 & - & 1 \\
\hline total & & $43 \mid$ & 12 & 30 & 25 & 25 & 47 & 241 & 71 & 620 & 1.130 & 555 & 596 & 3.178 \\
\hline
\end{tabular}

$45,0 \mathrm{~cm}$ de comprimento zoológico (tabelas IV $\mathrm{e} \mathrm{V}$; figura 1) .

Fizemos a distribuição dos tamanhos de indivíduos de cada espécie considerada, nos diversos conjuntos mensais de amostras (tabelas II a V; figura 2). A pesca da cavala, nos meses de abril a setembro, foi feita às custas de cardumes compostos dos grupos intermediários de idades, justamente quando as pescarias, em geral, foram levadas a efeito em águas mais próximas da costa, e que nos meses de janeiro a março e de outubro a dezembro, cardumes compostos de maior número de grupos de idades foram atingidos pela pesca, quando as pescarias se afastaram mais da costa. A pesca da serra, nos meses de janeiro a agôsto atingiu cardumes de composição, quanto aos grupos de idade, mais ou menos semelhantes, o mesmo não acontecendo no período de setembro a dezembro, quando atingiu um menor número de grupos de idades, muito semelhantes. Segundo Klima (1959), nas pescarias da serra ao sul da Flórida (U.S.A.), os cinco primeiros grupos de idades são os que compõem os desembarques. 
T A B E L A V

Freqüências relativas de serra, Scomberomorus maculatus (Mitchill, 1815), por classes de comprimento zoológico $(\mathrm{cm})$, por meses e no total das amostras. Material capturado em frente ao município de Fortaleza (Estado do Ceará - Brasil), durante os meses de janeiro a dezembro de 1966 .

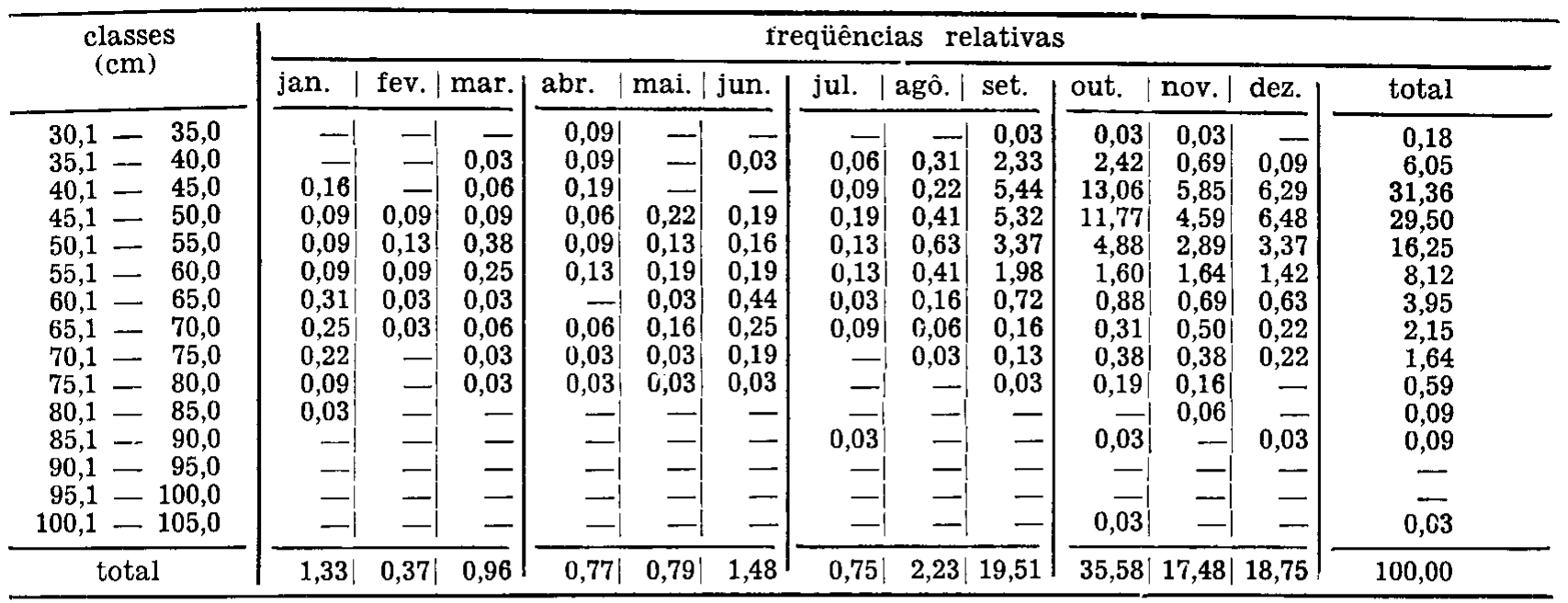

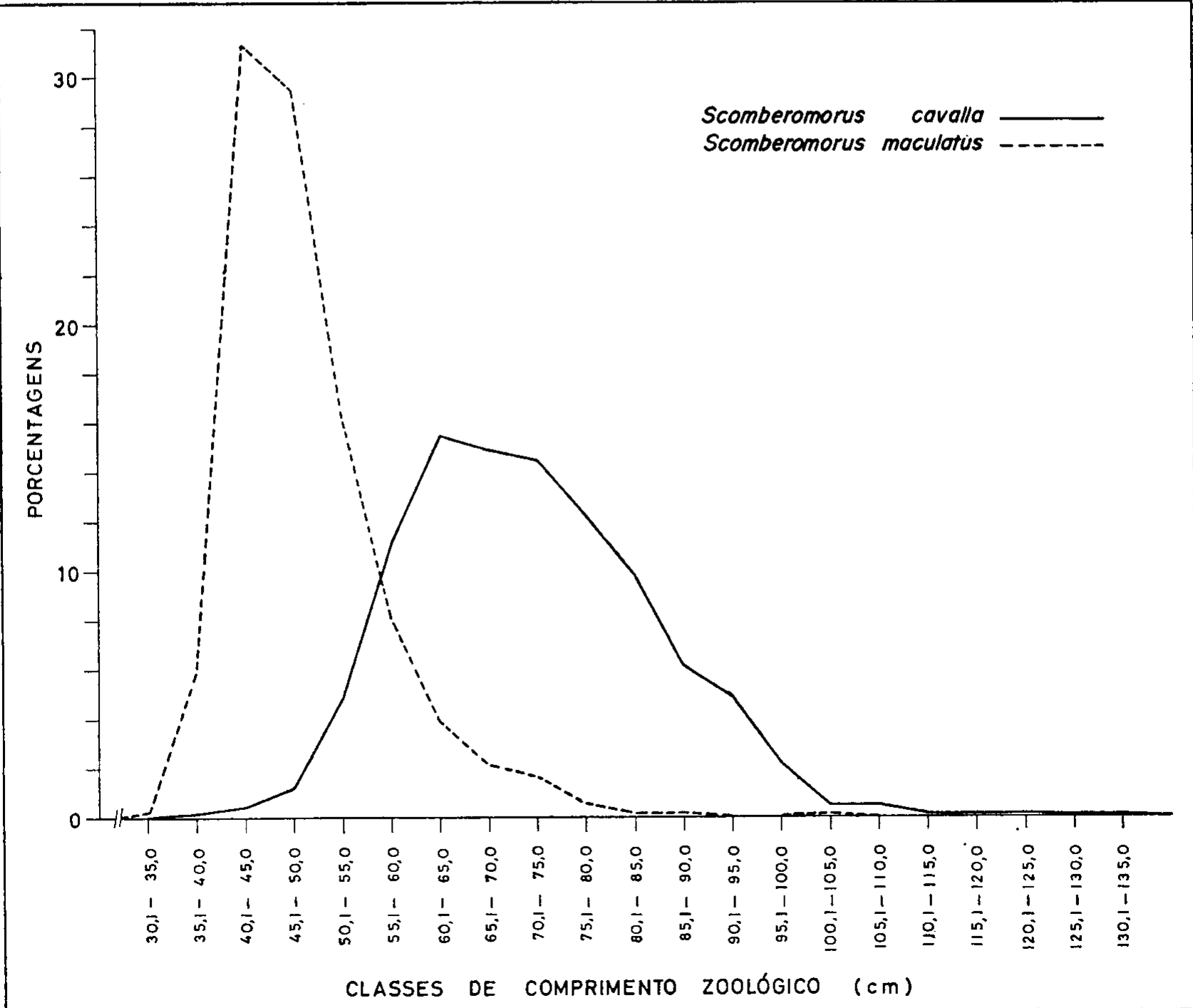

Figura 1 - Freqüências relativas de indivíduos das espécies Scomberomorus cavalla (Cuvier, 1829) e Scomberomorus maculatus (Mitchill, 1815), por classes de comprimento zoológico, no conjunto geral das amostras. Material capturado em frente ao município de Fortaleza (Estado do Ceará - Brasil), no período de $1 .^{\circ}$ de janeiro a 31 de dezembro de 1966 . 


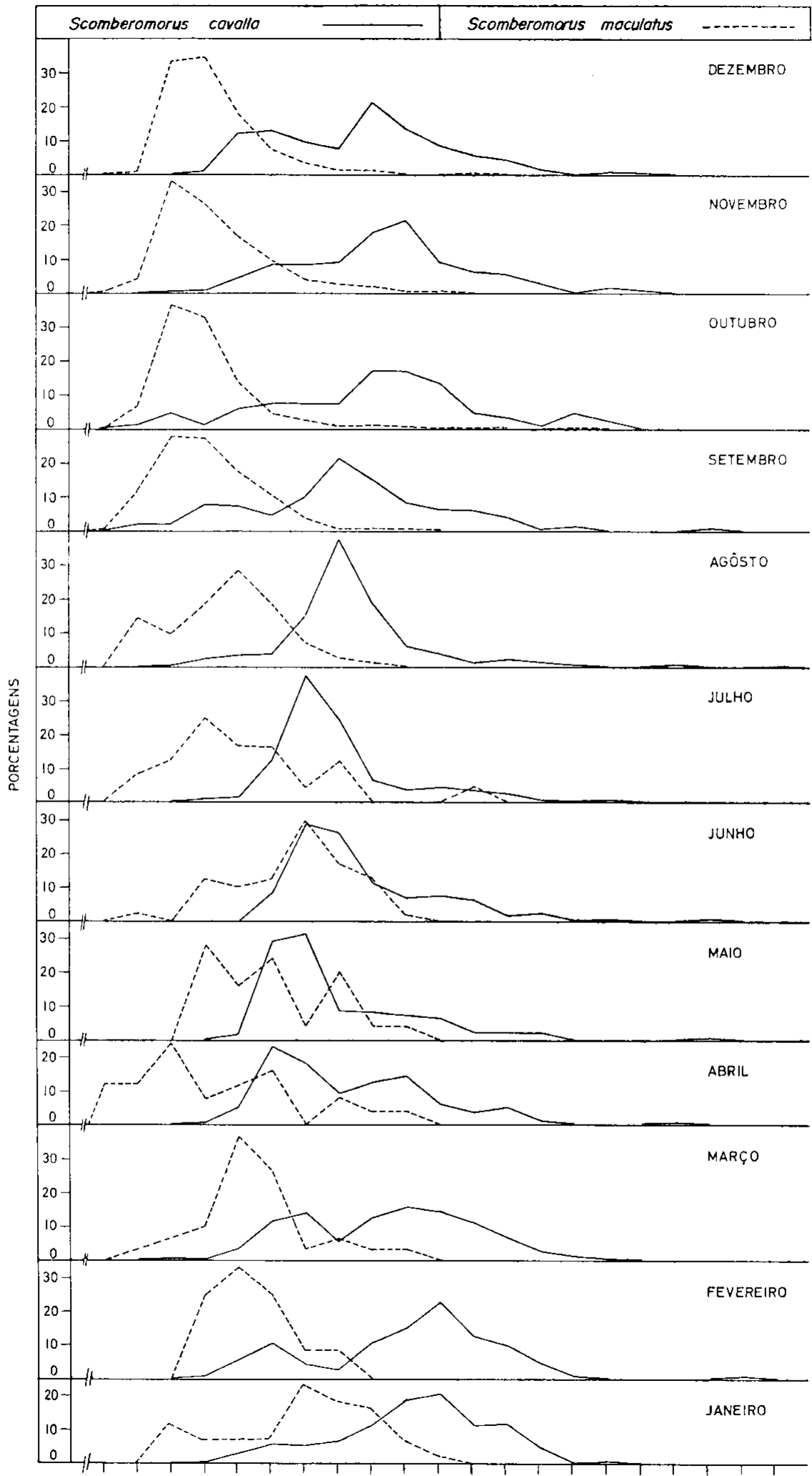

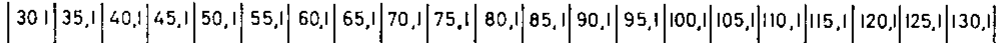

$\mid 35,0\} 40,0|45,0| 50,0|55,0| 60,0|65,0| 70,0|75,0| 80,0|85,0| 90,0|95,0| 100,0|105,0| 110,0|| 15,0|120,0| 125,0|130,0| 135,0 \mid$

CLASSES DE COMPRIMENTO ZOOLÓGICO $(\mathrm{cm})$

Figura 2 - Freqüências relativas de indivíduos das espécies Scomberomorus cavalla (Cuvier, 1829) e Scomberomorus maculatus (Mitchill, 1815) por classes de comprimento zoológico e nos diversos conjuntos mensais de amostras. Material capturado em frente ao município de Fortaleza (Estado do Ceará - Brasil), no período de $1^{\circ}$ de janeiro a 31 de dezembro de 1966 . 
Apesar do número de indivíduos amostrados de cada espécie em estudo ter variado muito entre os meses, as médias aritméticas dos comprimentos zoológicos correspondentes, bem como seus desvios padrões e coeficientes de variação são muito próximos (tabelas VI e VII ; figura 3) . Para a cavala, a maior destas médias foi a do mês de janeiro, quando atingiu c valor de $78,4 \mathrm{~cm}$ e a menor correspondeu ao mês de maio, expressa pelo valor de $67,0 \mathrm{~cm}$. Em relação à serra, a maior destas médias foi a do mês de janeiro, quando atingiu o valor de $62,5 \mathrm{~cm}$, e a menor correspondeu ao mês de outubro, expressa pelo valor de $47,2 \mathrm{~cm}$. Na tabela VIII apresentamos as médias dos comprimentos zoológicos correspondentes aos anos de idade da cavala e da serra, de acôrdo com Nomura \& Rodrigues (1967) e Nomura (1967), respectivamente.

O estudo comparativo das médias mensais de comprimentos zoológicos, tanto da cavala como da serra, referentes aos anos de 1962 a 1966 , com base nos trabalhos anteriores desta série (Costa \& Paiva, 1963 , 1964,1965 e 1966) e nos dados da presente contribuição, mostra a pouca semelhança existente entre os anos, não permitindo conhecer uma tendência geral bem evidente, na distribuição das referidas médias em ciclos anuais. Nota-se no entanto, que as médias mensais da cavala, em geral, são elevadas no primeiro e quarto trimestres, quandr. existem boas condições de navegação e as pescarias podem ser feitas em águas mais distantes da costa.

O número de pescarias controladas apesar de ter variado muito entre os meses, foi mais uniforme do que o do ano anterior (Costa \& Paiva, 1966). As menores freqüências mensais das pescarias controladas corresponderam aos meses de abril e julho, e as maiores, aos meses de março e dezembro (tabela IX) .

\section{T A B E L A V I}

Dados referentes aos comprimentos zoológicos (cm) de cavala, Scomberomorus cavalla (Cuvier, 1829). Material capturado em frente ao município de Fortaleza (Estado do Ceará — Brasil), durante os meses de janeiro a dezembro de 1966 .

\begin{tabular}{|c|c|c|c|c|c|c|}
\hline \multirow[b]{2}{*}{ meses } & \multirow[b]{2}{*}{$\begin{array}{l}\text { número } \\
\text { de } \\
\text { indivíduos } \\
\text { (n) }\end{array}$} & \multicolumn{5}{|c|}{ comprimento zoológico $(\mathrm{cm})$} \\
\hline & & $\begin{array}{l}\text { valor } \\
\text { máximo }\end{array}$ & $\begin{array}{l}\text { valor } \\
\text { mínimo }\end{array}$ & $\begin{array}{l}\text { média } \\
\text { aritmética } \\
\overline{(\bar{x})}\end{array}$ & $\begin{array}{l}\text { desvio } \\
\text { padrão } \\
\text { (s) }\end{array}$ & $\begin{array}{c}\text { coeficiente de } \\
\text { variação } \\
\text { (C.V.) }\end{array}$ \\
\hline $\begin{array}{l}\text { janeiro } \\
\text { fevereiro } \\
\text { março } \\
\text { abril } \\
\text { maio } \\
\text { junho } \\
\text { julho } \\
\text { agôsto } \\
\text { setembro } \\
\text { outubro } \\
\text { novembro } \\
\text { dezembro }\end{array}$ & $\begin{array}{r}270 \\
121 \\
283 \\
181 \\
182 \\
230 \\
178 \\
339 \\
189 \\
82 \\
204 \\
406\end{array}$ & $\begin{array}{l}110,0 \\
126,5 \\
108,7 \\
119,3 \\
122,6 \\
121,7 \\
109,8 \\
132,7 \\
120,8 \\
106,8 \\
114,7 \\
115,0\end{array}$ & $\begin{array}{l}49,3 \\
48,7 \\
44,8 \\
50,0 \\
51,5 \\
55,8 \\
45,4 \\
40,7 \\
37,3 \\
39,3 \\
41,8 \\
46,3\end{array}$ & $\begin{array}{l}78,4 \\
77,4 \\
75,0 \\
69,1 \\
67,0 \\
70,2 \\
67,1 \\
70,0 \\
68,9 \\
73,2 \\
74,2 \\
70,4\end{array}$ & $\begin{array}{r}11,71 \\
13,63 \\
12,55 \\
11,77 \\
11,32 \\
10,50 \\
9,65 \\
11,27 \\
14,25 \\
15,34 \\
12,65 \\
12,40\end{array}$ & $\begin{array}{l}14,94 \\
17,61 \\
16,73 \\
17,03 \\
16,90 \\
14,96 \\
14,38 \\
16,10 \\
20,68 \\
20,96 \\
17,05 \\
17,61\end{array}$ \\
\hline
\end{tabular}

T A B E L A V I I

Dados referentes aos comprimentos zoológicos (cm) de serra, Scomberomorus maculatus (Mitchill, 1815) . Material capturado em frente ao município de Fortaleza (Estado do Ceará - Brasil), durante os meses de janeiro a dezembro de 1966 .

\begin{tabular}{|c|c|c|c|c|c|c|}
\hline \multirow[b]{2}{*}{ meses } & \multirow[b]{2}{*}{$\begin{array}{l}\text { número } \\
\text { de } \\
\text { indivíduos } \\
\text { (n) }\end{array}$} & \multicolumn{5}{|c|}{ comprimento zoológico $(\mathrm{cm})$} \\
\hline & & $\begin{array}{l}\text { valor } \\
\text { máximo }\end{array}$ & $\begin{array}{l}\text { valor } \\
\text { mínimo }\end{array}$ & $\begin{array}{c}\text { média } \\
\text { aritmética } \\
\overline{(\bar{x})}\end{array}$ & $\begin{array}{c}\text { desvio } \\
\text { padrão } \\
\text { (s) }\end{array}$ & $\begin{array}{c}\text { coeficiente } \\
\text { de variação } \\
\text { (C.V.) }\end{array}$ \\
\hline $\begin{array}{l}\text { janeiro } \\
\text { fevereiro } \\
\text { março } \\
\text { abril } \\
\text { maio } \\
\text { junho } \\
\text { julho } \\
\text { agôsto } \\
\text { setembro } \\
\text { outubro } \\
\text { novembro } \\
\text { dezembro }\end{array}$ & $\begin{array}{r}43 \\
12 \\
30 \\
25 \\
25 \\
47 \\
24 \\
71 \\
620 \\
1.130 \\
555 \\
596\end{array}$ & $\begin{array}{r}81,2 \\
68,4 \\
78,4 \\
76,9 \\
79,2 \\
77,5 \\
88,6 \\
70,3 \\
77,3 \\
102,1 \\
84,7 \\
85,8\end{array}$ & $\begin{array}{l}41,5 \\
45,3 \\
39,9 \\
33,3 \\
45,8 \\
35,2 \\
38,2 \\
36,6 \\
31,6 \\
33,9 \\
31,8 \\
37,9\end{array}$ & $\begin{array}{l}62,5 \\
55,4 \\
55,1 \\
49,4 \\
57,7 \\
60,7 \\
53,9 \\
50,8 \\
47,8 \\
47,2 \\
49,3 \\
48,4\end{array}$ & $\begin{array}{r}11,01 \\
6,80 \\
8,16 \\
12,33 \\
8,74 \\
8,83 \\
11,14 \\
7,86 \\
6,96 \\
6,70 \\
8,24 \\
6,37\end{array}$ & $\begin{array}{l}17,62 \\
12,27 \\
14,81 \\
24,96 \\
15,15 \\
14,55 \\
20,67 \\
15,47 \\
14,56 \\
14,56 \\
16,71 \\
13,16\end{array}$ \\
\hline
\end{tabular}




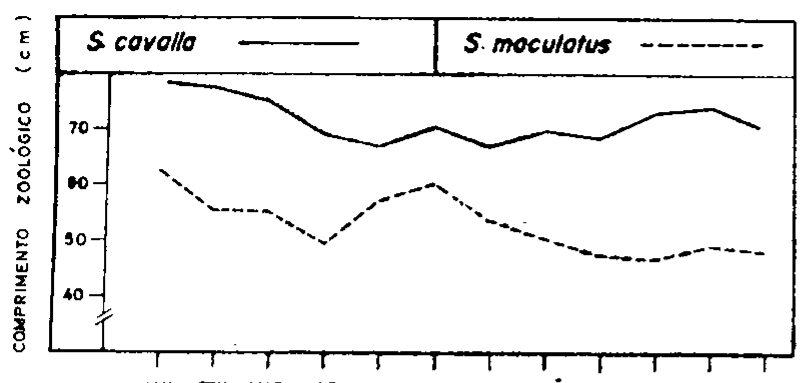

meses -1900

Figura 3 - Distribuição das médias aritméticas de comprimentos zoclógicos de indivíduos das espécie Scomberomorus cavalla (Cuvier, 1829) e Scomberomorus maculatus (Mitchill, 1815), correspondentes aos diversos conjuntos mensais de amostras. Material capturado em frente ao município de Fortaleza (Estado do Ceará - Brasil), no período de $1 .^{\circ}$ de janeiro a 31 de dezembro de 1966 .

\section{T A B E L A V I I I}

Médias de comprimentos zoológicos (cm) correspondentes aos anos de idade de cavala, Scomberomorus cavalla (Cuvier, 1829), e de serra, Scomberomorus maculatus (Mitchill, 1815), capturadas em frente à costa do Estado do Ceará - Brasil, de acôrdo com Nomura \& Rodrigues (1967) e Nomura (1967), respectivamente.

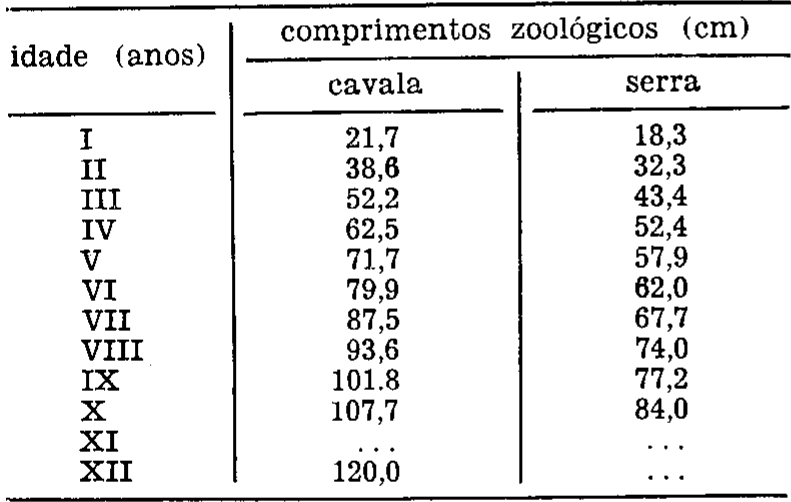

Os totais de anzóis/dia empregados nas pescarias controladas, em geral, variaram em relação direta com as freqüências mensais das pescarias. As médias mensais do número de anzóis utilizados por pescaria, se apresentaram mais ou menos constantes. A média anual de anzóis por pescaria foi de 5,4 anzóis. Ressaltamos que os tipos de anzóis, freqüentemente usados nas pescarias controladas, foram os de número 4 e 5 para a cavala, e os de número 6 e 7 para a serra.

Os tipos de isca usados nas pescarias controladas, foram a sardinha - Opisthonema oglinum (Le Sueur, 1818), a xira - Haemulon aurolineatum Cuvier, 1829 e a (s) traíra (s) - Trachinocephalus myops (Forster, 1801) $\mathrm{e} / \mathrm{ou}$ Synodus intermedius (Agassiz, 1828), em ordem decrescente de importância, sendo também usados outros pequenos peixes marinhos, em escala muito pequena, podendo

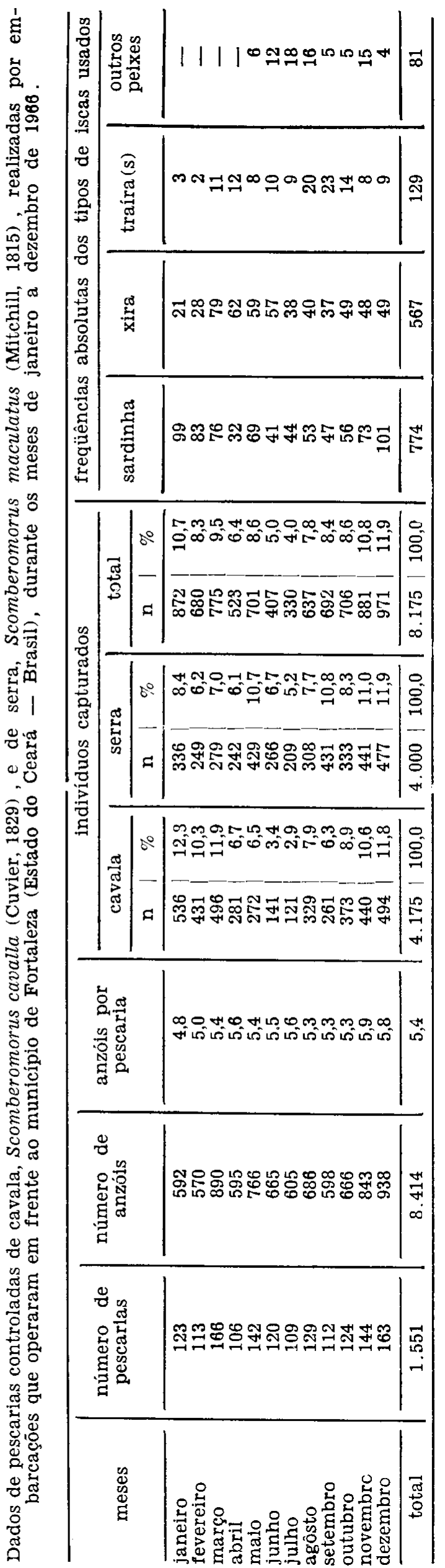


mesmo não serem tomados em consideração. As freqüências mensais dos tipos de iscas usados nas pescarias, mostram que nos meses de janeiro, fevereiro e maio e no período de julho a dezembro, a sardinha foi mais usada do que os demais tipos, tendo predominado a xira nos meses de março, abril e junho (tabela IX) .

Nas pescarias controladas sòmente foram consideradas as capturas de cavala e de serra, que atingiram um total de 8.175 indivíduos, sendo 4.175 cavalas e 4.000 serras. As maiores capturas mensais da cavala ocorreram nos períodos de janeiro a março e novembro a dezembro, e as de serra, nos meses de maio, setembro, novembro e dezembro. Já em relação ao total dos indivíduos capturados em tais pescarias, observamos que as maiores capturas mensais corresponderam aos meses de janeiro, novembro e dezembro (tabela IX) .
Estudamos as relações número de indivíduos capturados/número de pescarias e números de indivíduos capturados por 100 anzóis/dia (densidade relativa), referentes a cada mês e ao ano, e também a cada espécie e para ambas em conjunto (tabela $\mathbf{X}$; figura 4). Os índices determinados permitem a delimitação de safras, que entendemos como as épocas de maior disponibilidade da (s) população(ões), para pescarias com embarcações, artes e métodos pesqueiros tradicionalmente usados na área em estudo, que coincidem com os maiores índices de densidade relativa. Tanto para a cavala, como para a serra, como para ambas em conjunto, os mais elevados índices de densidade relativa corresponderam aos meses de janeiro e fevereiro e ao período de setembro a dezembro. Estas informações sôbre safras, que ora apresentamos, em parte concordam com as existentes nos

T A B E L A X

fndices mensais de captura por pescaria e de densidade relativa (captura por 100 anzóis/dia) de cavala, Scomberomorus cavalla (Cuvier, 1829), e de serra, Scomberomorus maculatus (Mitchill, 1815), na área de Fortaleza (Estado do Ceará — Brasil), e pluviosidade na mesma área, durante os meses de janeiro a dezembro de 1966 .

\begin{tabular}{|c|c|c|c|c|c|c|c|}
\hline \multirow[t]{2}{*}{ meses } & \multicolumn{3}{|c|}{$\frac{\text { número de indivíduos capturados }}{\text { número de pescarias }}$} & \multicolumn{3}{|c|}{$\begin{array}{l}\text { densidade relativa } \\
\text { (captura por } 100 \text { anzóis/dia) }\end{array}$} & \multirow[t]{2}{*}{$\begin{array}{l}\text { pluviosidade } \\
(\mathrm{mm})\end{array}$} \\
\hline & cavala & serra & total & cavala & serra & total & \\
\hline $\begin{array}{l}\text { janeiro } \\
\text { fevereiro } \\
\text { março } \\
\text { abril } \\
\text { maio } \\
\text { junho } \\
\text { juiho } \\
\text { agôsîo } \\
\text { setembro } \\
\text { outubro } \\
\text { novembro } \\
\text { dezembro }\end{array}$ & $\begin{array}{l}4,4 \\
3,8 \\
3,0 \\
2,7 \\
1,9 \\
1,2 \\
1,1 \\
2,6 \\
2,3 \\
3,0 \\
3,1 \\
3,0\end{array}$ & $\begin{array}{l}2,7 \\
2,2 \\
1,7 \\
2,3 \\
3,0 \\
2,2 \\
1,9 \\
2,4 \\
3,8 \\
2,7 \\
31 \\
2,9\end{array}$ & $\begin{array}{l}7,1 \\
6,0 \\
4,7 \\
5,0 \\
4,9 \\
3,4 \\
3,0 \\
5,0 \\
6,1 \\
5,7 \\
62 \\
5,9\end{array}$ & $\begin{array}{l}90,5 \\
75,6 \\
55,7 \\
47,2 \\
35,5 \\
21,2 \\
20,0 \\
48,0 \\
43,6 \\
56,0 \\
52 . ? \\
52,7\end{array}$ & $\begin{array}{l}56,8 \\
43,7 \\
31,3 \\
40,7 \\
56,0 \\
40,0 \\
34.5 \\
44,9 \\
72,1 \\
50,0 \\
52,3 \\
50,9\end{array}$ & $\begin{array}{r}147,3 \\
119,3 \\
87,0 \\
87,9 \\
91,5 \\
61,2 \\
54,5 \\
92,9 \\
115,7 \\
106,0 \\
104,5 \\
103,6\end{array}$ & $\begin{array}{r}27,2 \\
48,2 \\
130,9 \\
329,3 \\
300,5 \\
154,3 \\
156,4 \\
15,7 \\
44,6 \\
2,0 \\
19,4 \\
9,8\end{array}$ \\
\hline total & 2,7 & 2,6 & 5,3 & 49,6 & 47.5 & 97,1 & $1.288,3$ \\
\hline
\end{tabular}

* deixaram de ser consideradas as outras espécies capturadas.

Obs.: Os dados sôbre a pluviosidade foram fornecidos pela Estação Metereológica de Fortaleza, pertencente ao Serviço de Meteorologia do Ministério da Agricultura do Brasil.

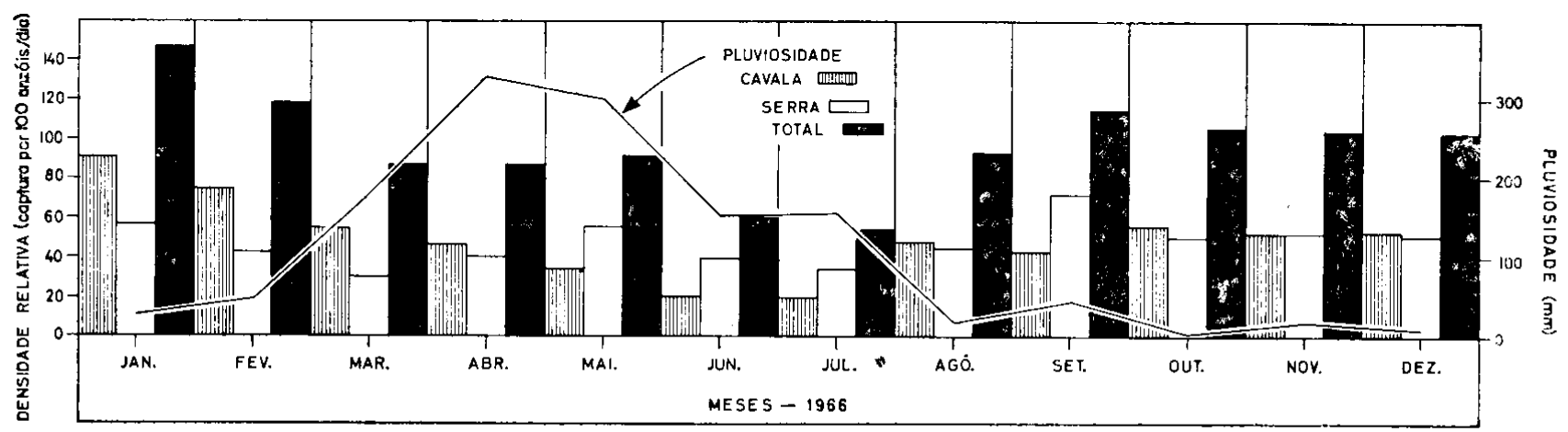

Figura 4 - Distribuição dos índices de densidade relativa (captura por 100 anzóis/dia) das espécies $S c o m$ beromorus cavalla (Cuvier, 1829) e Scomberomorus maculatus (Mitchill, 1815), de ambas as espécies em conjunto, e da pluviosidade na área em estudo. Material capturado em frente ao município de Fortaleza (Estado do Ceará - Brasil), no periodo de $10^{\circ}$ de janeiro a 31 de dezembro de 1966 . 
trabalhos anteriores da série (Costa \& Paiva, $1963,1964,1965$ e 1966).

Procuramos encontrar alguma relação entre a densidade relativa das espécies consideradas e a pluviosidade, na área em estudo (tabela $\mathrm{X}$; figura 4) . Em geral, para a cavala, a relação em referência foi indireta nos meses de janeiro a abril, direta para o período de maio a julho e irregular para os demais meses do ano. Já para a serra, esta relação se mostrou indireta para o primeiro trimestre e irregular para os demais meses do ano. Considerando ambas as espécies em conjunto, a relação foi indireta no primeiro trimestre e irregular para os demais trimestres do ano.

\section{S U M M A R Y}

With this paper the Marine Biology Station of the Federal University of Ceará carries on a serial study on the fishery biology of the king mackerel, Scomberomorus cavalla (Cuvier, 1829) and Spanish mackerel, Scomberomorus maculatus (Mitchill, 1815) in the State of Ceará, Brazil.

The material was caught off Fortaleza, and landed at Mucuripe Beach. It corresponds to sample of 234 days of fishing carried out during period from January to December, 1965 , made by rafts and some motor boats. We sampled 2,665 king mackerels and 3,178 Spanish mackerels. We have also controlled 1,551 raft fishings, in the same area and throughout the months from January to December, 1965 .

For the king mackerel, the fork length reached by the fishery, in the set of samples, ranged from 35.0 to $135.0 \mathrm{~cm}$, being observed concentration between 50.0 and $95.0 \mathrm{~cm}$, and maximum between 60.0 and $65.0 \mathrm{~cm}$. The catches reached individuals from II to XII years old. It seems that the older king mackerels live farther from the coast than the younger ones.

For the Spanish mackerel the fork lengths reached by the fishery, in the set of samples, ranged from 30.0 to $110.0 \mathrm{~cm}$, being observed concentration between 35.0 and $60.0 \mathrm{~cm}$, and maximum between 40.0 and $45.0 \mathrm{~cm}$. The catches reached individuals from II to more than $\mathrm{X}$ years old.

In the several monthly set of samples, the arithmetic means of fork lengths corresponding to each one of the species, as well as its standard deviations and variation coefficients are very close. For the king mackerel the greatest among those arithmetic means corresponded to the month of January, when reached the value of $78.4 \mathrm{~cm}$, and the smallest one was reported in the month of May, expressed through the value of $67.0 \mathrm{~cm}$. For the Spanish mackerel, the greatest among those arithmetic means corresponded to the month of January, when reached the value of $62.5 \mathrm{~cm}$, and the smallest one was reported in the month of October, expressed through the value of $47.2 \mathrm{~cm}$.

The total number of hooks monthly used in controlled fishing, in general, varied in direct relationship to the monthly frequencies of fishing. The monthly means of the number of hooks used in each fishing were more or less constant. The year mean of hooks in each fishing was 5.4 hooks.

The types of bait used in controlled fishing were the Atlantic thread - herring Opisthonema oglinum (Le Sueur, 1818), the tomtate - Haemulon aurolineatum Cuvier, 1829 and snakefish - Trachinocephalus myops (Forster, 1801) and/or sand diver Synodus intermedius (Agassiz, 1828) in de. creasing order of importance, being also used other marine little fish, in lesser number.

In the controlled fishing were considered only the king mackerel and Spanish mackerel catches, that have reached to a total of 8,175 individuals, being 4,175 king mackerels and 4,000 Spanish mackerels.

We have studied the relations number of caught individual number of fishing and number of individuals caught by 100 hooks / day (relative density). The numbers found permit us to determine the harvest. These were coincident with the periods of highest relative density. The highest numbers of relative density for the king mackerel as for the Spanish mackerel, and for both, were in the months of January and February, and in the period from September to December.

\section{BIBLIOGRAFIA CONSULTADA}

Costa, R. S. \& Paiva, M. P. - 1963 - Notas sôbre a pesca da cavala e da serra no Ceará - Dados de 1962 . Arq. Est. Biol. Mar. Univ. Ceará, Fortaleza, 3 (1) : 17-26, 4 figs.

Costa, R. S. \& Paiva, M. P. - 1964 - Notas sôbre a pesca da cavala e da serra no Ceará - Dados de 1963. Arq. Est. Biol. Mar. Univ. Ceará, Fortaleza, 4 (2): $71-81,5$ figs.

Costa, R. S. \& Paiva, M. P. - 1965 - Notas sôbre a pesca da cavala e da serra no Ceará - Dados de 1964. Arq. Est. Biol. Mar. Univ. Ceará, Fortaleza, 5 (2) : 93-101, 5 figs.

Costa, R. S. \& Paiva, M. P. - 1966 - Notas sôbre a pesca da cavala e da serra no Ceará - Dados de 1965. Arq. Est. Biol. Mar. Univ. Fed. Ceará, Fortaleza, 6 (2) : 195-204, 4 figs.

Fowler, H. W. - 1941 - A list of the fishes know from the coast of Brazil. Arq. Zool. Est. São Paulo, São Paulo, 3 (6) : 115-184.

Fraser-Brunner, A. - 1950 - The fishes of the Family Scombridae. Ann. Mag. Nat. Hist., London, ser. 12,3 (26) : 131-163, 35 figs.

Klima, E. F. - 1959 - Aspects of the Biology and the fishery for Spanish mackerel, Scomberomorus maculatus (Mitchill), of Southern Florida. State of Florida Board of Conservation Technical Series, Coral Gables, (27) : 1-39, 17 figs. 
Nomura, H. 1967 - Dados biológicos sôbre a serra, Scomberomorus maculatus (Mitchill), das águas cearenses. Arq. Est. Biol. Mar. Univ. Fed. Ceará, Fortaleza, 7 (1) : 29-39, 4 figs.

Nomura, H. \& Rodrigues, M. S. S. - 1967 Biological notes on king mackerel, Scomberomorus cavalla (Cuvier), from northeastern Brazil. Arq. Est.
Biol. Mar. Univ. Fed. Ceará, Fortaleza, 7 (1) : 79-85, 4 figs.

Rivas, L. R. - 1951 - A preliminary Review of the Western North Atlantic Fishes of the Family Scombridae. Bull. Mar. Sci. Gulf Caribb., Coral Gables, 1 (3) : 209-230. 